\title{
Contact Force Radiofrequency Ablation: Is It the Gold Standard for Atrial Fibrillation Ablations?
}

\author{
MOUSSA MANSOUR, MD ${ }^{1}$ \\ ${ }^{1}$ Heart Center, Massachusetts General Hospital, Boston, Massachusetts
}

\begin{abstract}
Contact force sensing is a relatively new technology, and its use has been rapidly expanding. This paper provides an overview of the most recent studies in this field and explains why this technology has been so effective. It presents data from the SMART-AF and TOCCASTAR studies, describes the use of force sensing for the assessment of lesion formation, and discusses the economic impact of using contact force radiofrequency ablation for atrial fibrillation ablation.
\end{abstract}

KEYWORDS. contact force sensing, atrial fibrillation, contact force radiofrequency, atrial fibrillation ablation, paroxysmal atrial fibrillation.
ISSN 2156-3977 (print) ISSN 2156-3993 (online)

(C) 2016 Innovations in Cardiac Rhythm Management

\section{SMART-AF and TOCCASTAR}

SMART-AF and TOCCASTAR are probably the two most important studies in the field of force sensing. This is a brief summary of the two studies.

SMART-AF was a prospective, multicenter, non-randomized study. ${ }^{1}$ The test device was the THERMOCOOL ${ }^{\mathbb{R}}$ SMARTTOUCH ${ }^{\mathrm{TM}}$ device (Biosense Webster, Inc., South Diamond Bar, CA), and its safety and efficacy were compared with predetermined performance goals. The study was conducted at 21 centers in the United States, and 172 patients with paroxysmal atrial fibrillation were enrolled. They were treated with pulmonary vein isolation ablation (PVI).

The TOCCASTAR study was a prospective, multicenter, randomized study with a non-inferiority design comparing


with a control device, the Navistar ${ }^{\mathrm{TM}}$ Thermocool $^{\mathrm{TM}}$ (Biosense Webster, Inc., South Diamond Bar, CA). The study was performed at 17 sites in the United States and Europe. In total, 300 patients were enrolled. These patients were the same population as for the SMART-AF; they all had paroxysmal atrial fibrillation treated with PVI.

Dr. Mansour is a consultant for Biosense-Webster, St. Jude Medical, Medtronic, and Boston Scientific. Dr. Mansour has received research grants from St. Jude Medical, Biosense-Webster, Boston Scientific, and MC10.

Manuscript received March 8, 2016, final version accepted April 4, 2016.

Address correspondence to: Moussa Mansour, M.D., Heart Center, Massachusetts General Hospital, 55 Fruit Street, Boston, MA 02114. E-mail: mmansour@partners.org

\section{Endpoints}

In SMART-AF, a non-randomized study, the primary effectiveness endpoint was met (Figure 1), and the success rate was about $74 \%$ at the 12 -month follow-up. The study included $9.2 \%$ of patients who were on Class I/III anti-arrhythmia drugs; two subjects were on new anti-arrhythmia drugs (AAD) during follow-up, and were deemed protocol failures.

The primary effectiveness endpoint in TOCCASTAR, a non-inferiority design study, was also met (Figure 2). There were two definitions for treatment success: protocol-defined success (left) and clinically relevant treatment success (right). With either definition, the treatment/test catheter (TactiCath) was non-inferior to the control catheter (Navistar Thermocool).

There are two important points that should be borne in mind. First, SMART-AF and TOCCASTAR had different definitions of success, so their success rates cannot be compared. Second, how contact force was used in these two studies is a critical point to understand the results, and this is discussed further below.

\section{Use of contact force in the studies}

The parameters of contact force in SMART-AF and TOCCASTAR are as follows. Per protocol, the physicians were not required to use a specific amount of contact force in either study; this was left to the discretion of the operator. In SMART-AF, the operators selected their force 


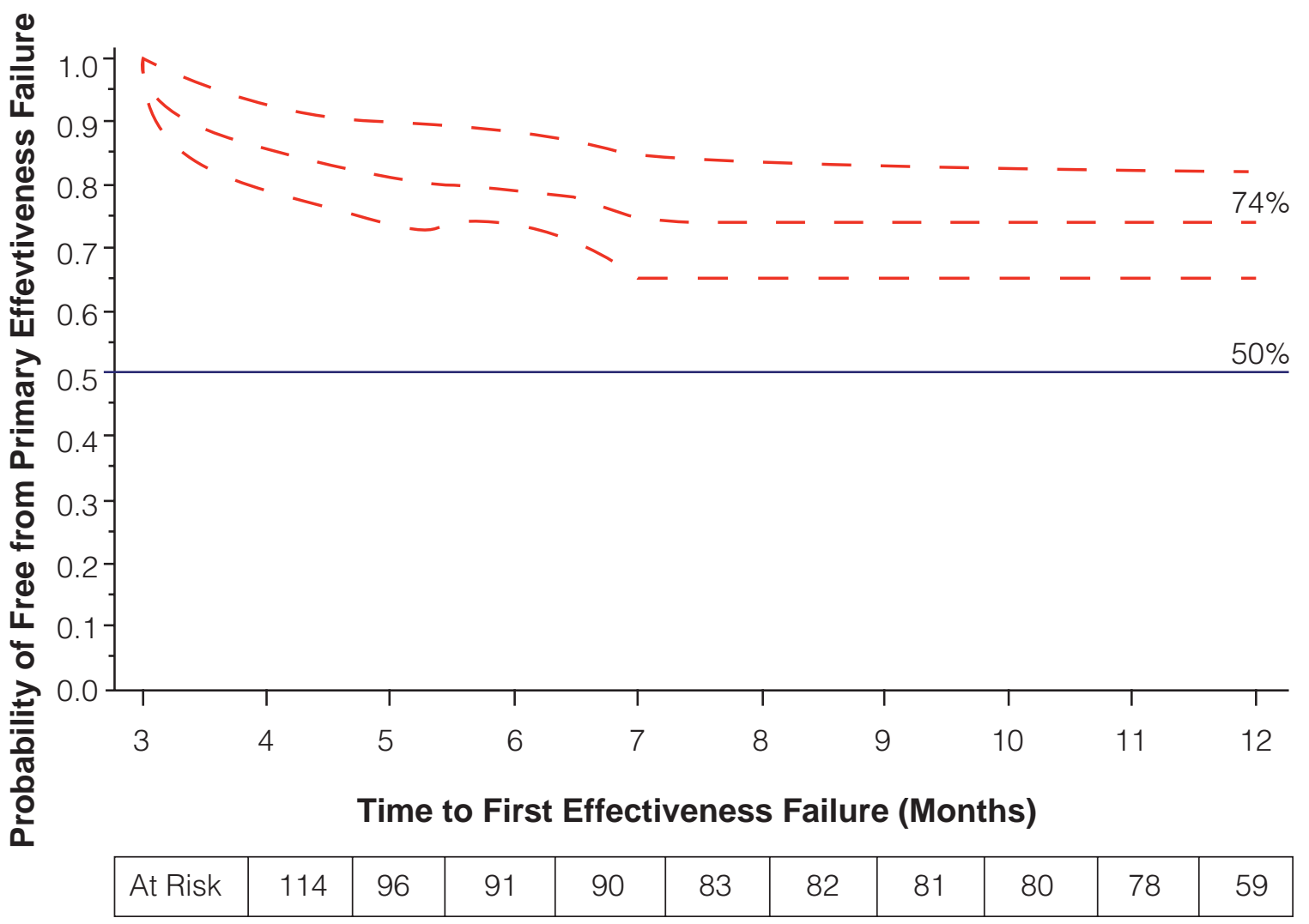

Figure 1: Primary effectiveness in SMART-AF ${ }^{1} .9 .2 \%$ of patients were on Class I/III AAD includes 2 subjects who were on new $A A D$ during follow-up and were deemed protocol failures. Reprinted from Journal of the American College of Cardiology, 64/7, Andrea Natale, MD, Vivek Y. Reddy, MD, George Monir, MD, et al, Paroxysmal AF Catheter Ablation With a Contact Force Sensing Catheter Results of the Prospective, Multicenter SMART-AF Trial, Pages 647-656, 2014, with permission from Elsevier under CC BY-NC-ND 4.0. (doi:10.1016/j.jacc.2014.04.072).

working range, and a post hoc analysis was performed to study the effect of percentage of time the operator stayed in the selected range. In TOCCASTAR, some operators used a contact force of $>10 \mathrm{~g}$, and a prespecified analysis was used to study the effect of optimal force, which was defined as $\geq 90 \%$ of the lesion being ablated at $\geq 10 \mathrm{~g}$ of force. ${ }^{3,4}$

In SMART-AF, the percentage of time the operators stayed in the selected force range was assessed, whereas in TOCCASTAR the study assessed how often the operators used optimal force. In SMART-AF, operators remained in their preselected force range $73 \%$ of the time. In TOCCASTAR, $57 \%$ of the patients were treated with optimal force, and $43 \%$ of the patients were treated with suboptimal force. These results are not comparable between the two studies, because of the the definition of "optimal force" was not the same. However, both studies show a very important factor, in that a significant number of patients were not treated with optimal force, because that was required by protocol.

Figure 3 shows the impact of staying in the preselected force range in SMART-AF. If the operator stayed in their selected force range more than $80 \%$ of the time (orange curve), the success rate was $82 \%$, compared with just $65 \%$ if they stayed within the range $<80 \%$ of the time. This was not only the case at this time percentage $(80 \%)$; this was studied across the wider percentages. As shown in Figure 3, which is a plot of success rate versus percentage of time spent in the selected force ranges during ablation, the important finding is that the greater the time the operator stayed within the selected range, the higher the success rate.

This was also consistent with the findings from TOCCASTAR, which showed that there was an improved success rate when ablation was performed at optimal force.

\section{Use of force sensing for the assessment of lesion formation}

Lesion assessment is probably the most important unmet need in electrophysiology. In the SMART-AF and TOCCASTAR studies, force information was not integrated with other ablation parameters such as duration, power, and catheter stability, which are needed to assess lesion formation. However, technology has moved on since SMART-AF and TOCCASTAR completed enrolment.

\section{New-generation mapping system that came after TOCCASTAR}

Similarly, a new-generation mapping system was introduced subsequent to the TOCCASTAR study. It is a mathematical model integrating data from force, duration, and power. Similar to the system above, it integrates force with other inputs for ablation (duration and power), 

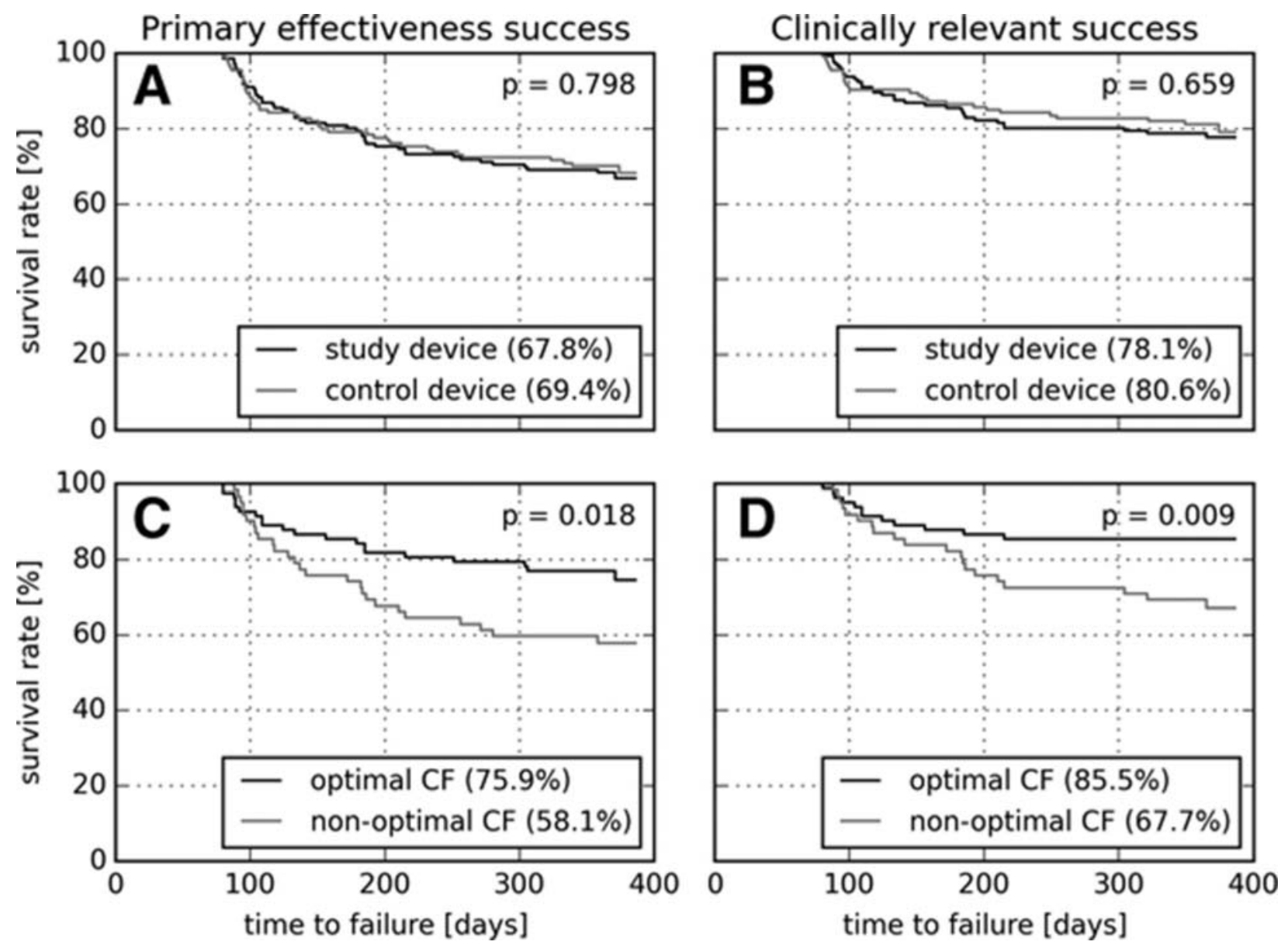

Figure 2: Treatment success in TOCCASTAR. Reprinted from Circulation, 132/10, Vivek Y. Reddy, Srinivas R. Dukkipati, Petr Neuzil, et al, Randomized, Controlled Trial of the Safety and Effectiveness of a Contact Force-Sensing Irrigated Catheter for Ablation of Paroxysmal Atrial Fibrillation, Pages 907-915, 2015, with permission from American Heart Association, Inc.

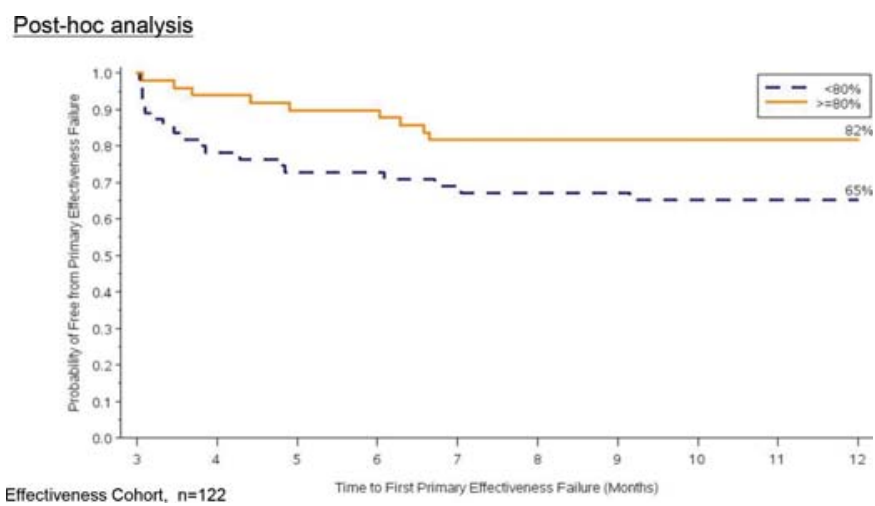

Figure 3: Effect of staying in the preselected force range in SMART-AF. Reprinted from Journal of the American College of Cardiology, 64/7, Andrea Natale, MD, Vivek Y. Reddy, MD, George Monir, MD, et al, Paroxysmal AF Catheter Ablation With a Contact Force Sensing Catheter Results of the Prospective, Multicenter SMART-AF Trial, Pages 647-656, 2014, with permission from Elsevier under CC BY-NC-ND 4.0. (doi:10.1016/j.jacc.2014.04.072).

producing an assessment of your lesion formation. This results in a lesion size index (LSI), which is shown as a number on screen and is very helpful in assessing lesion formation.

This system was tested retrospectively I the EFFICAS-I study, and showed that LSI correlates strongly with pulmonary vein isolation at 3 months. And EFFICAS-I is a study where patients were brought back for an electrophysiology study whether or not they had occurrence of atrial fibrillation to see if they had gaps. When the LSI was 5.7, there were no gaps, whereas when the LSI was lower, there were gaps. 


\section{Conclusion}

In summary, force sensing improves the efficacy of atrial fibrillation ablation when ablation is performed at optimal contact force.

- The integration of force sensing with other inputs such as catheter location, power, and duration is likely to allow accurate assessment of lesion formation.

- The most recent data shows the use of force sensing is associated with economic benefit.

\section{References}

1. Natale A, Reddy VY, Monir G, et al. Paroxysmal-AF catheter ablation with a contact force sensing catheter:
Results of the prospective, multicenter, SMART-AF trial. J Am Coll Cardiol. 2014;64(7):647-656.

2. Reddy VY, Dukkipati SR2, Neuzil P, et al. Randomized, controlled trial of the safety and effectiveness of a contact force-sensing irrigated catheter for ablation of paroxysmal atrial fibrillation: Results of the TactiCath contact force ablation catheter study for atrial fibrillation (TOCCASTAR) study. Circulation. 2015;132(10):907-915.

3. Reddy V, Shah D, Kautzner J, et al. The relationship between contact force and clinical outcome during radiofrequency catheter ablation of atrial fibrillation in the Toccata study. Heart Rhythm. 2012;9(11):1789-1795.

4. Neuzil P, Reddy V, Kautzner J, et al. Electrical reconnection after pulmonary vein isolation is contingent on contact force during initial treatment: results from the Efficas I Study. Circ Arrhythm Electrophysiol. 2013;6(2):327-333. 